\title{
Ventricular Rhythm and Hypotension in a Patient with Pheochromocytoma-induced Myocardial Damage and Reverse Takotsubo Cardiomyopathy
}

\author{
Minoru Tagawa ${ }^{1}$, Hitomi Nanba ${ }^{1}$, Hiromi Suzuki ${ }^{2}$, Yuichi Nakamura ${ }^{1}$, Hiroko Uchiyama ${ }^{1}$, \\ Sachie Ochiai ${ }^{1}$, Masahiro Terunuma ${ }^{3}$, Kazuaki Yahata ${ }^{2}$ and Tohru Minamino ${ }^{4}$
}

\begin{abstract}
A 33-year-old woman experienced near-syncope at a hospital. Electrocardiography revealed an intermittent ventricular rhythm. The echocardiogram at admission indicated mild hypokinesis and severe diffuse hypokinesis with reverse takotsubo cardiomyopathy on the following day. The patient experienced abdominal pain on the admission day, and computed tomography identified a large left adrenal mass. Her catecholamine levels increased remarkably on the third day. The wall motion improved on the twelfth day. The tumor was successfully resected and the patient was diagnosed with an ectopic pheochromocytoma. The ventricular rhythm with myocardial damage and hypotension induced by the reverse takotsubo cardiomyopathy masked the characteristic symptoms of pheochromocytoma.
\end{abstract}

Key words: ventricular rhythm, hypotension, myocardial damage, reverse takotsubo cardiomyopathy, pheochromocytoma

(Intern Med 54: 2343-2349, 2015)

(DOI: 10.2169/internalmedicine.54.4732)

\section{Introduction}

Pheochromocytoma is a rare type of catecholaminesecreting tumor that presents with the classic symptoms of headache, diaphoresis, and tachycardia with paroxysmal hypertension $(1,2)$. Atypical presentations of pheochromocytoma, such as catecholamine-induced cardiomyopathy, myocardial ischemia, cardiac arrhythmia, or congestive heart failure, have also been reported (3-5). In the present report, we describe the case of a 33-year-old woman who presented with hypotension, bradycardia, and transient cardiomyopathy, or "reverse takotsubo" and was eventually diagnosed with pheochromocytoma.

\section{Case Report}

A 33-year-old woman presented to our hospital with a 2- week history of headache, palpitations, chest pain, general fatigue and dyspnea. She had undergone a 6-day treatment course for pneumonia at our hospital 1 month earlier and had undergone an appendectomy at 10 years of age. She reported no episodes of syncope or cardiac disease and had no family history of syncope or hormonal diseases. Her body weight had been maintained at approximately $57 \mathrm{~kg}$ for several years and her appetite and bowel movement were normal before she presented to our hospital. However, she exhibited near-syncope at the hospital and an electrocardiogram (ECG) revealed an intermittent ventricular rhythm with a heart rate of 60 beats per minute (Fig. 1A) and an inverted $\mathrm{T}$ wave in the V1-V3 leads during the sinus rhythm at a heart rate of 72 beats per minute (Fig. 1B). Her blood pressure was $102 / 58 \mathrm{mmHg}$ and her body temperature was normal. The hematological and serological examination results demonstrated an elevated leukocyte count $(10,780 \mathrm{u} / \mathrm{L})$ and positive troponin T. However, the creatine kinase $(\mathrm{CK})$ and

\footnotetext{
${ }^{1}$ Department of Cardiology, Nagaoka Chuo General Hospital, Japan, ${ }^{2}$ Department of Endocrinology, Nagaoka Chuo General Hospital, Japan, ${ }^{3}$ Department of Urology, Nagaoka Chuo General Hospital, Japan and ${ }^{4}$ Department of Cardiovascular Biology and Medicine, Niigata University Graduate School of Medical and Dental Sciences, Japan

Received for publication December 17, 2014; Accepted for publication February 1, 2015

Correspondence to Dr. Minoru Tagawa, m-tagawa@dc5.so-net.ne.jp
} 
(A)

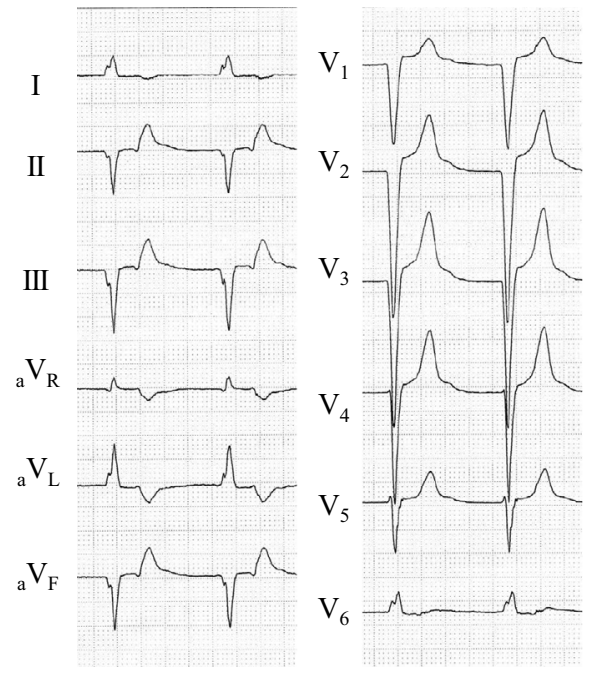

(B)

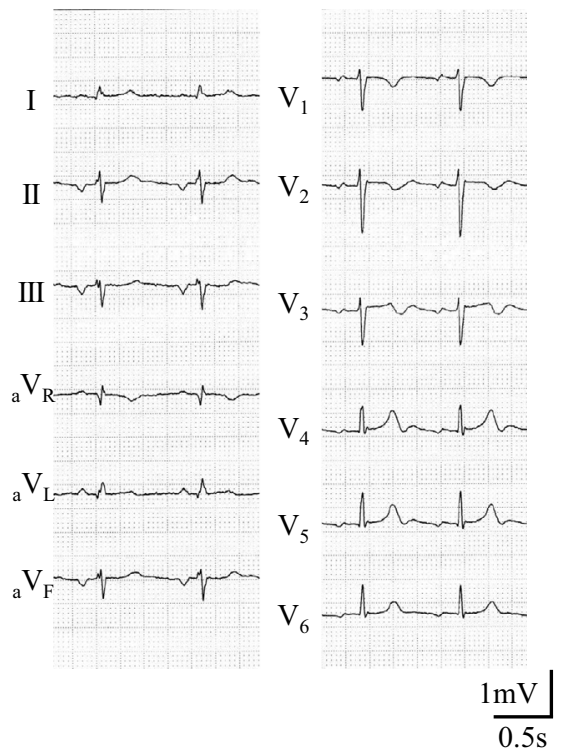

Figure 1. Electrocardiograms (ECGs) obtained on admission. A: Initial ECG during the ventricular rhythm with a heart rate of 60 beats per minute. B: ECG during the sinus rhythm showing the inverted $T$ waves in the V1-V3 leads.

(A)

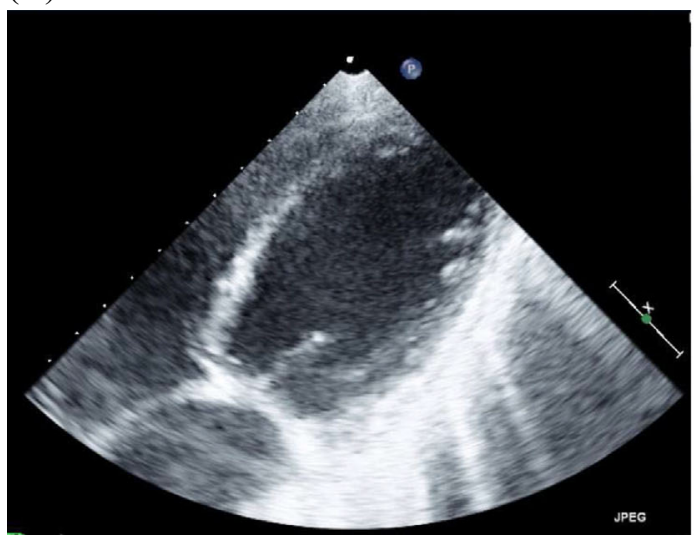

(B)

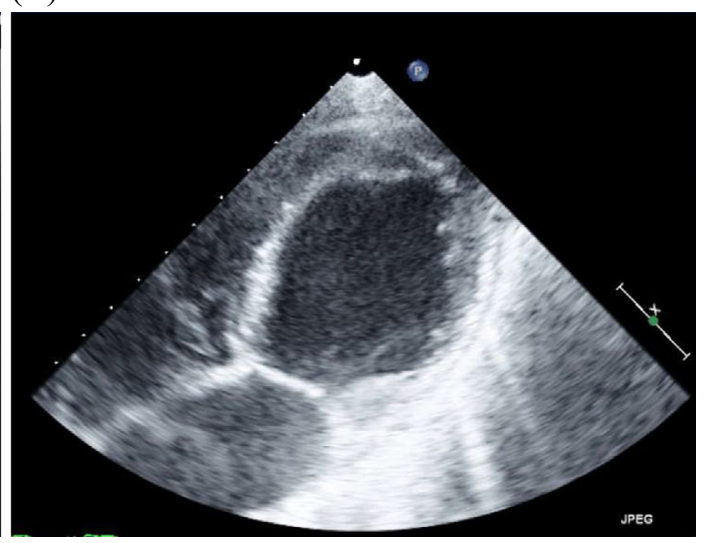

Figure 2. The apical four-chamber view of the patient during left ventricular diastole (A) and systole (B) on the day after admission. Echocardiography revealed severe diffuse hypokinesis except for in the apex and basal wall with a left ventricular ejection fraction of $22 \%$.

CK-MB levels were within the normal ranges (CK: $145 \mathrm{IU} /$ $\mathrm{L}$ and CK-MB: $17 \mathrm{IU} / \mathrm{L})$. The chest radiography findings were also normal. Two-dimensional echocardiography demonstrated a normal-sized left ventricle (LV) and slight hypokinesis of the middle portion of the anterior and anteroseptal wall with an ejection fraction (EF) of $69 \%$. The patient was then admitted to our hospital.

She briefly experienced abdominal pain at midnight on the day of admission. The day after admission, her systolic blood pressure was decreased to $70-90 \mathrm{mmHg}$. Twodimensional echocardiography revealed severe diffuse hypokinesis except for in the apex and basal wall and an EF of $22 \%$, which was suggestive of reverse takotsubo cardiomyopathy (Fig. 2). The ECG performed during the sinus rhythm on the day after admission showed an increased in the heart rate to 99 beats per minute and an inverted $\mathrm{T}$ wave in the V2-V6 leads (Fig. 3). The chest radiography findings revealed the appearance of lungs congestion (Fig. 4). The patient's CK and CK-MB levels had also increased [creatine phosphokinase (CPK): 258 IU/L and CK-MB: 35 IU/L].

Computed tomography (CT) was performed to investigate the abdominal pain and revealed a round $40 \mathrm{~mm} \times 40 \mathrm{~mm}$ lesion with well-defined margins in the left abdomen (Fig. 5A). Abdominal echocardiography also determined that the mass lesion comprised multiple nonhomogeneous, cystic lesions (Fig. 5B). These results suggested an extra-adrenal pheochromocytoma.

After obtaining informed consent, we performed catheterization. The right ventricular pressure and LV end diastolic pressure (EDP) were evaluated [pulmonary artery $(\mathrm{PA})=36$ / 

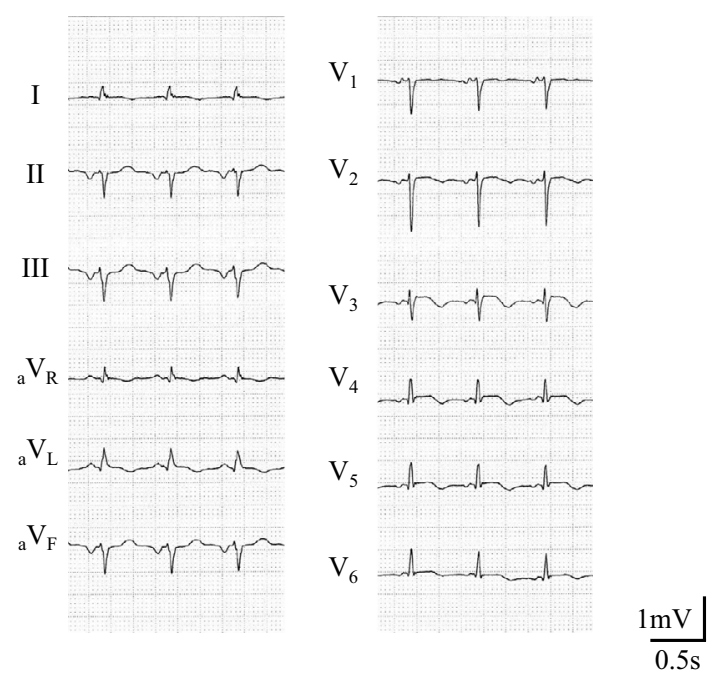

Figure 3. An Electrocardiogram (ECG) performed on the day after admission. ECG during the sinus rhythm showing the inverted $\mathrm{T}$ waves in the V2-V6 leads.

20 (27) $\mathrm{mmHg}$; LV = 133/EDP $35 \mathrm{mmHg}$. Coronary angiography found no remarkable coronary stenosis. Left ventriculography showed a depressed LVEF of $32.4 \%$ with severe middle portion ventricular hypokinesis (Fig. 6). Therefore, a temporary pacing device was inserted.

The patient was treated with milrinone $(0.5 \mu \mathrm{g} / \mathrm{kg} / \mathrm{min})$ for hypotension and the LV dysfunction. Two-dimensional echocardiography revealed that the LV wall motion had improved to an EF of $63 \%$ on the twelfth day after admission (Fig. 7). The inverted $\mathrm{T}$ waves in the V2-V6 leads had also normalized, the heart rate had decreased to approximately 54 beats per minute, and the ventricular rhythms frequencies decreased after the initiation of treatment (Fig. 8A). The chest radiography findings was also improved to within the normal range after the treatment (Fig. 8B).

The levels of adrenaline, noradrenaline, metanephrine, normetanephrine and vanillylmandelic acid (VMA) in the patient's urine were remarkably elevated on the third day after admission (Table 1). Similarly, the serum levels of adrenaline, noradrenaline, and dopamine were increased to $1.68 \mathrm{ng} / \mathrm{mL}, 18.31 \mathrm{ng} / \mathrm{mL}$, and $0.04 \mathrm{ng} / \mathrm{mL}$, respectively, on the day after admission. Adrenal scintigraphy found an ${ }^{123} \mathrm{I}-$ meta-iodobenzylguanidine (MIBG) accumulation only within the abdominal mass. As these findings were compatible with pheochromocytoma, an alpha-adrenergic blocking agent was administered (doxazosin mesilate $0.5 \mathrm{mg} /$ day up to $4 \mathrm{mg}$ / day) after the blood pressure had improved. The urinary levels of adrenaline, noradrenaline, metanephrine, normetanephrine and VMA peaked on the third day after admission and subsequently decreased (Table 1). The LV functions were also improved (Table 2).

After providing informed consent, the patients underwent successful surgical tumor resection while in a stable physical condition on the 45th day after admission. The tumor measured $40 \times 30 \times 25 \mathrm{~mm}$ and comprised both solid and necrotic bleeding lesions (Fig. 9). The levels of adrenaline, norad-

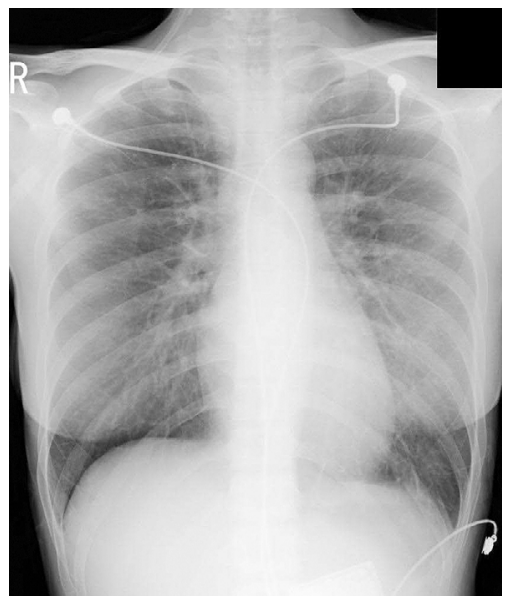

Figure 4. Chest radiography performed on the day after admission. Chest radiography reveals the appearance of bilateral lungs congestion.

renaline, and dopamine in the necrotic lesion exudate were $30.14 \mathrm{ng} / \mathrm{mL}, 284.36 \mathrm{ng} / \mathrm{mL}$, and $0.34 \mathrm{ng} / \mathrm{mL}$, respectively, and a pathological assessment confirmed the presence of ectopic pheochromocytoma. All of the urinary catecholamine levels normalized thereafter (Table 1).

The patient has not experienced syncope since undergoing beta-blocker therapy after the surgical treatment.

\section{Discussion}

In the present case, both the ventricular rhythms associated with myocardial injury and hypotension induced by reverse takotsubo cardiomyopathy masked the characteristic symptoms of pheochromocytoma, a rare catecholaminesecreting tumor of the neuroendocrine system $(1,2)$. The classic symptoms of this type of tumor include hypertension, hypermetabolism, hyperglycemia, headache, and hyperhidrosis (1). However, at admission, the patient was nearsyncope and the ECG revealed an intermittent ventricular rhythm that made it difficult to diagnose the pheochromocytoma.

Pheochromocytoma has been shown to present as myocardial ischemia, tachyarrhythmia, congestive heart failure, and cardiac symptoms that can be associated with hypotension and a fluctuating blood pressure, but the frequency of shock is low (4-6). The mechanism of hypotension may explain how excess norepinephrine can induce myocardial injury and the blood vessel desensitization thereby leading to the adrenergic stimulation of the pheochromocytoma $(4,5,7)$. In the present case, although the ventricular rhythm and LV dysfunction of the anterior wall at admission may have been induced by the excess norepinephrine, no tachycardia or hypertension was induced.

Some case reports have also indicated that a pheochromocytoma can induce cardiomyopathy with a reverse takotsubo contractile pattern $(3,8-11)$. Reverse takotsubo cardiomyopathy has been reported as an LV dysfunction in which the basal and mid-ventricular segments are hypokinetic al- 
(A)

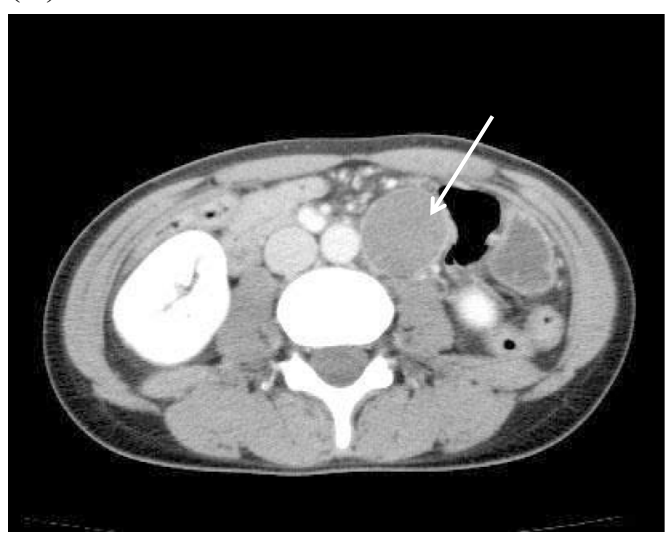

(B)

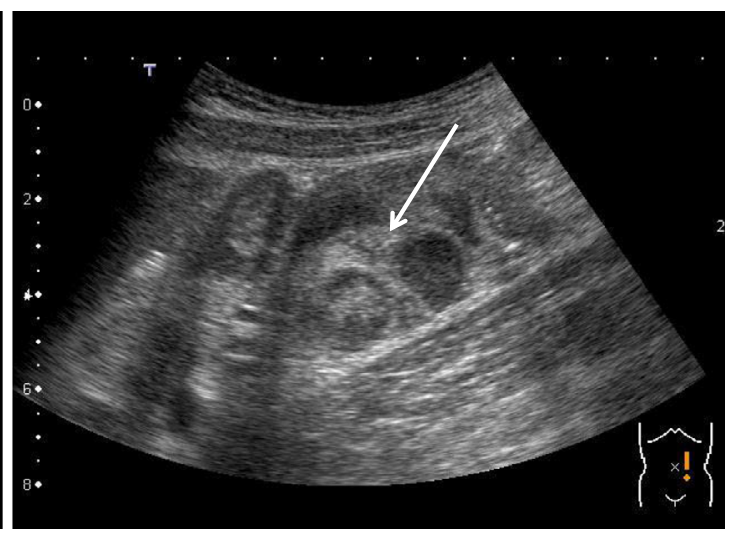

Figure 5. Pheochromocytoma. A: Computed tomography performed the day after admission to investigate abdominal pain revealed a round lesion measuring $40 \mathrm{~mm} \times 40 \mathrm{~mm}$ in the left abdomen. B: An abdominal echocardiogram revealed that the mass lesion was nonhomogeneous and included multiple cystic lesions suggestive of extra-adrenal pheochromocytoma.

(A)

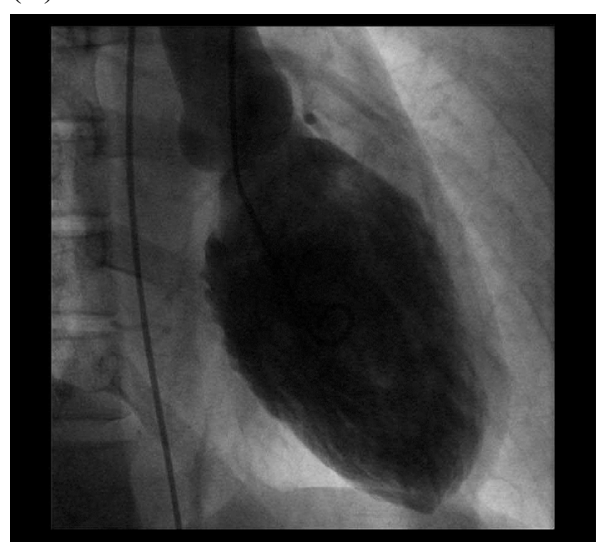

(B)

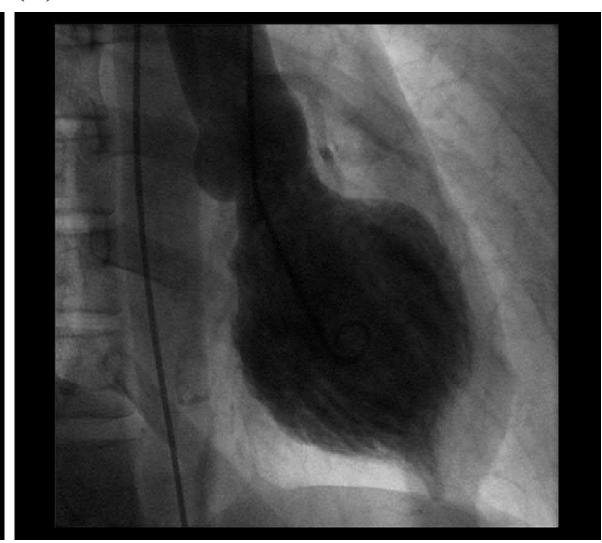

Figure 6. Left ventricle angiography performed the day after admission. Left ventricle angiography showed a depressed left ventricular ejection fraction of $32.4 \%$ with severe hypokinesis of the mid-ventricle. This result was compatible with reverse takotsubo cardiomyopathy.

(A)

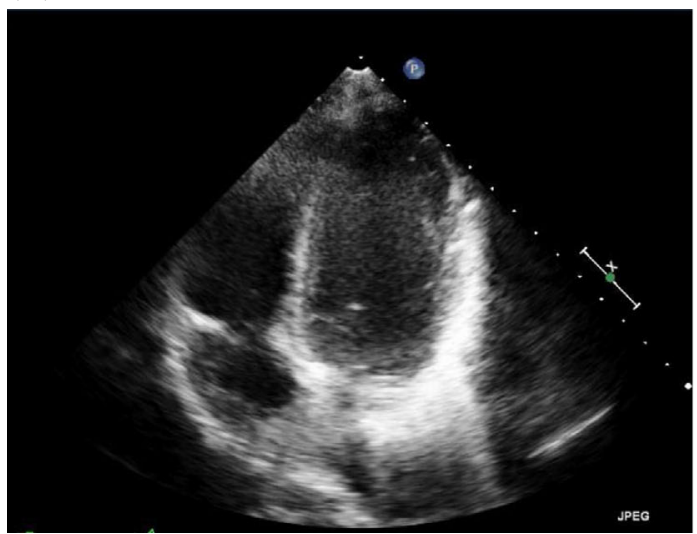

(B)

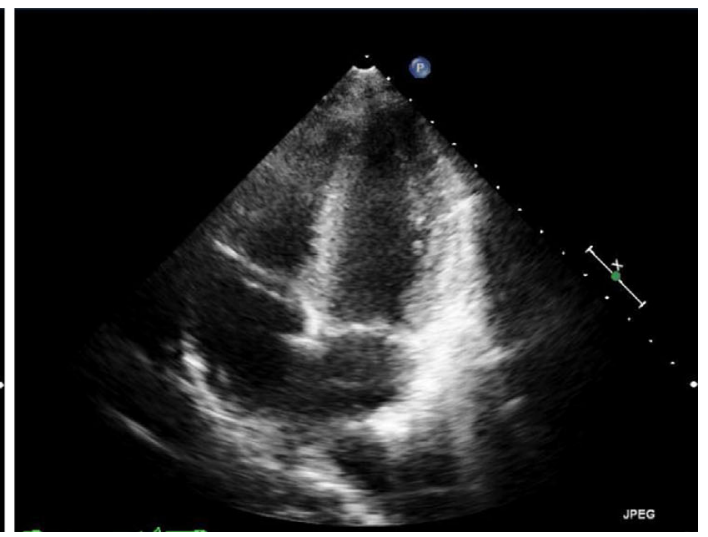

Figure 7. The apical four-chamber view of the patient during left ventricular diastole (A) and systole (B) after recovery. Echocardiography showed that the left ventricle wall motion improved within the normal range. 
(A)

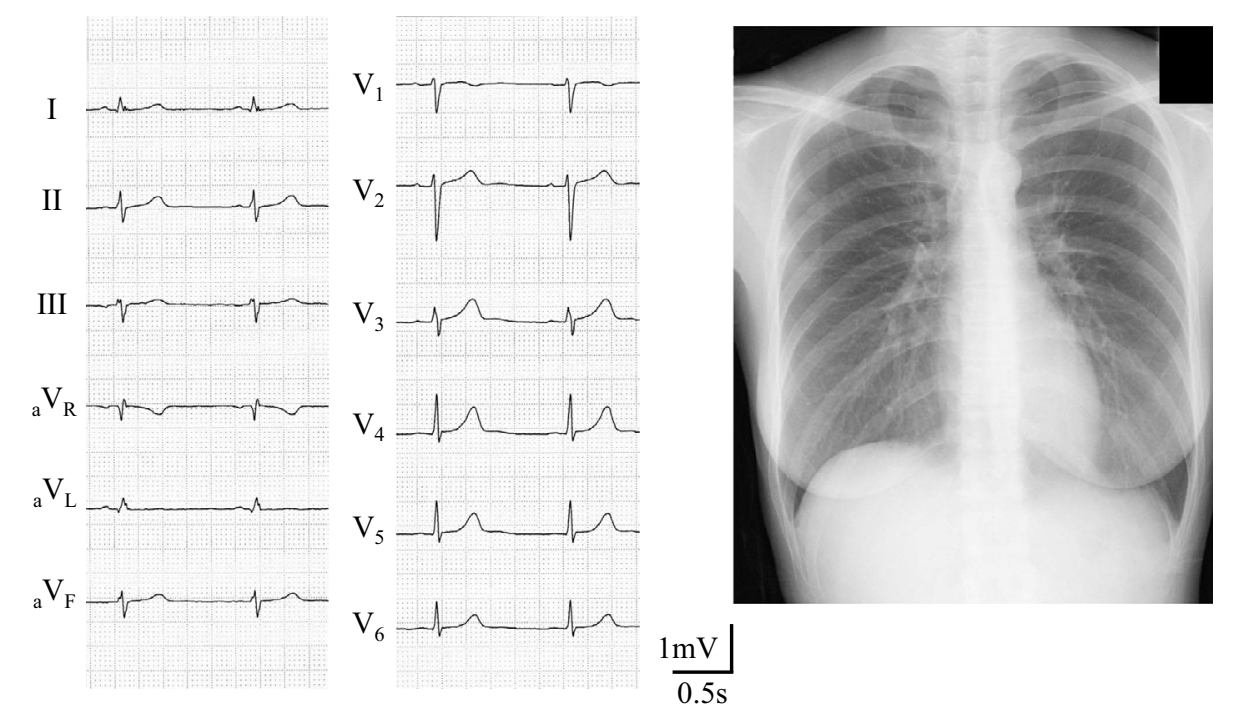

Figure 8. ECG and chest radiography taken after recovery. (A) The inverted T waves in the V2-V6 leads normalized and the heart rate decreased to 54 beats per minute. (B) Chest radiography showed a decrease in the cardiothoracic rate from 44.5 to $39.9 \%$ and the disappearance of lung congestion.

Table 1. Patient's Urinary Catecholamine Levels over Time. The Levels of Adrenaline, Noradrenaline, Metanephrine, Normetanephrine and Vanillylmandelic Acid (VMA) were Remarkably Elevated on the Day after Admission but Decreased after the Treatment.

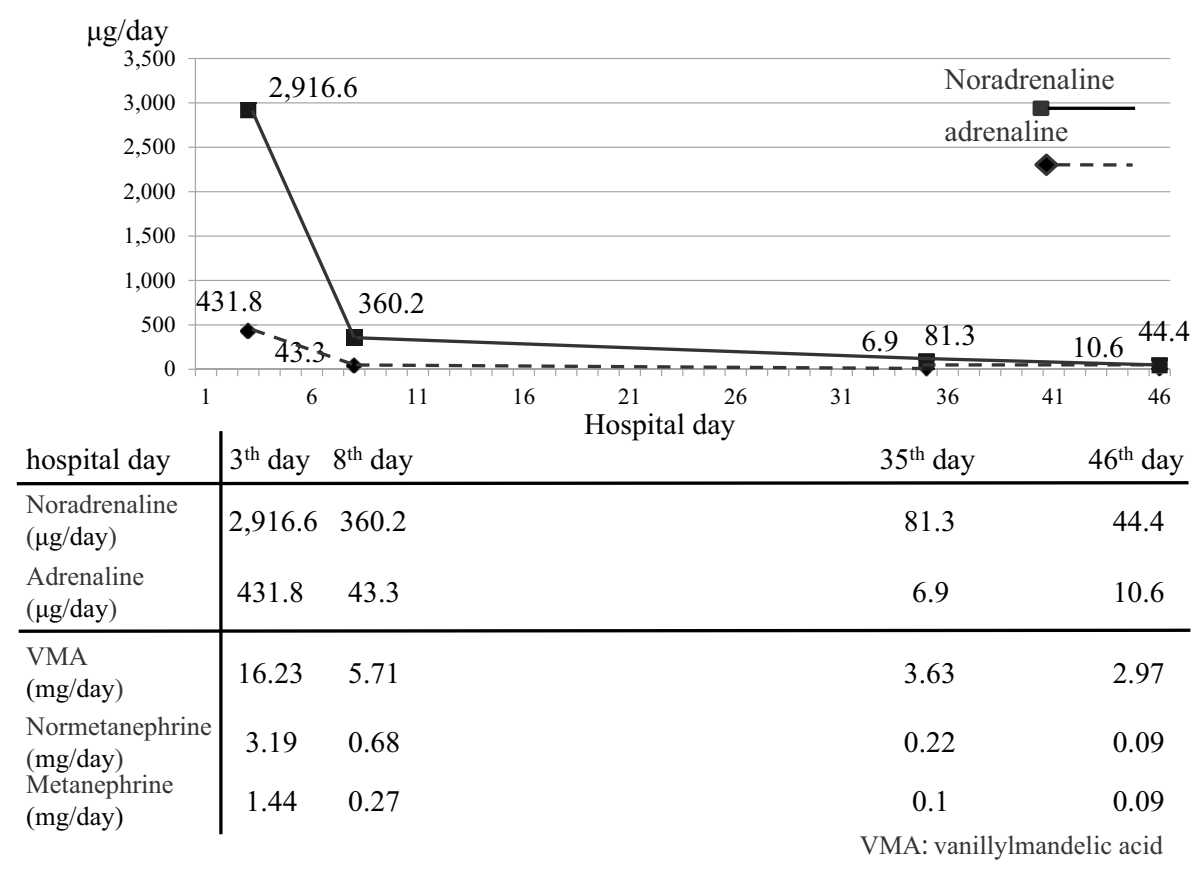

though the apex appears normal. In the present case, on the day after admission, two-dimensional echocardiography revealed severe diffuse hypokinesis except for in the apex and basal wall with an EF of $22 \%$, suggestive of reverse takotsubo cardiomyopathy. This condition worsened with the LV dysfunction. The disturbed conduction, LV dysfunction with myocardial injury, and reverse takotsubo cardiomyopathy induced the hypotension, thus masking the pheochromocytoma and rendering the diagnosis difficult.
Reverse takotsubo cardiomyopathy is considered a type of takotsubo or stress-induced cardiomyopathy (12-14). Takotsubo cardiomyopathy is characterized by a rapid and severe reversible cardiac dysfunction without significant coronary arterial stenosis (12). Emotional or physical stressors have been described as triggering factors of this syndrome (15). Four types of LV dysfunction in takotsubo cardiomyopathy have been described: classical, reverse, mid-ventricular, and local $(11,14,16)$. Regional differences in the adrenergic 
Table 2. The Ejection Fraction via Two-dimension Echocardiography over Time. Echocardiography on the Day after Admission Revealed Severe Diffuse Hypokinesis Except for in the Apex and Basal Wall with an Ejection Fraction of 22\%. The Left Ventricle Wall Motion Improved to within the Normal Range on the Twelfth Day after Admission.

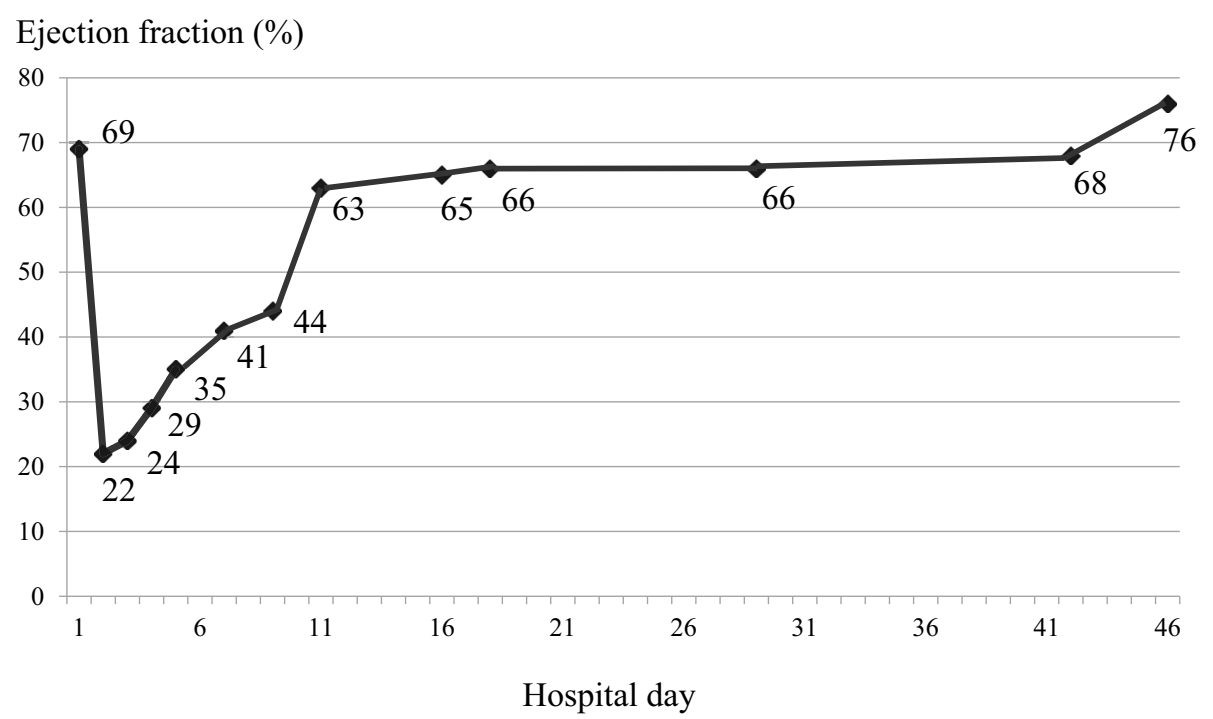

(A)

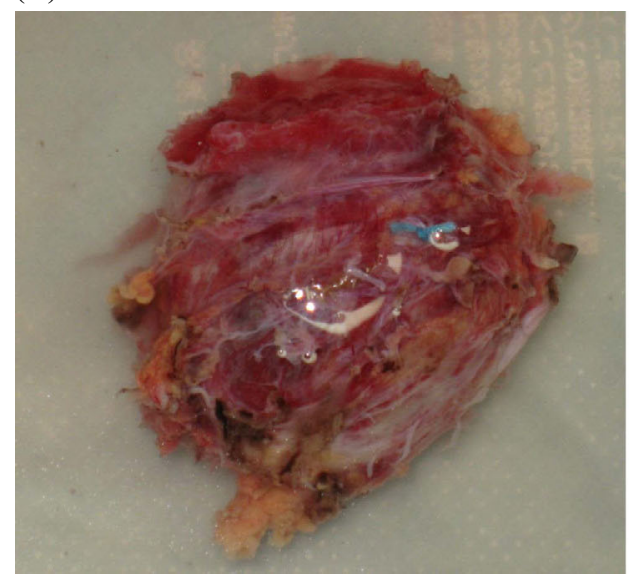

(B)

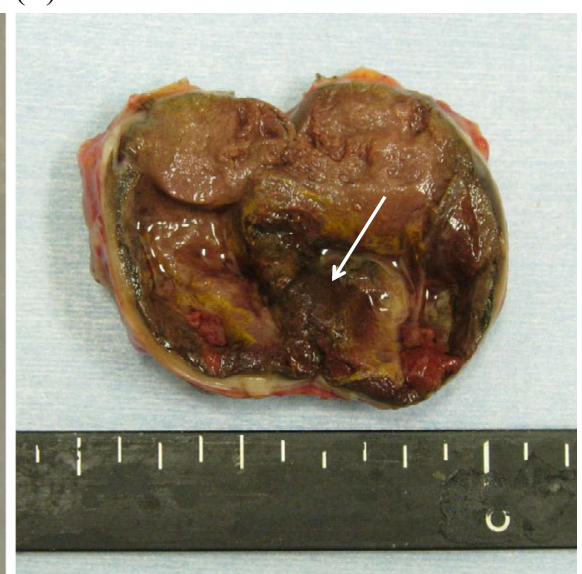

Figure 9. The tumor and ectopic pheochromocytoma. (A) The entire tumor. The tumor size was $40 \times 30 \times 25 \mathrm{~mm}$. (B) A Longitudinal section of the tumor. The tumor comprises both solid and necrotic bleeding lesions (arrow indicates the necrotic lesion). Elevated catecholamine levels were observed in the necrotic lesion exudate.

sensitivity or innervation may explain the differences in the clinical presentations compared with other regions of hypokinesis. In the present case, the patient had not experienced a significant triggering episode (e.g., emotional stress). Some reports have suggested that the presence of reverse takotsubo cardiomyopathy indicates the presence of a pheochromocytoma $(17,18)$. Moreover, our patient experienced abdominal pain on the night of admission. Thus, we believe the pheochromocytoma induced reverse takotsubo cardiomyopathy. Accordingly, it is important to examine the patients carefully and to cautiously present the differential diagnoses according to the various symptoms of pheochromocytoma.
The serum catecholamine levels are generally evaluated in the patients on the day after admission. As such, continuous dopamine/dobutamine or norepinephrine provocation may not facilitate the blood pressure evaluations or improve the LV function in the presence of such excessive catecholamine levels. Therefore, we used the milrinone to improve the patient's LV function and treat hypotension.

Therapy for pheochromocytoma typically includes the use of an adequate alpha-adrenergic blockade. However, as the LV dysfunction and hypotension were remarkable in the present case, we initiated treatment with a low-dose alphaadrenergic blocking agent after the LV function and blood pressure had improved and then carefully increased the 
dose. The surgical treatment was successful in the context of the alpha-adrenergic agent therapy.

According to a previous case report, necrosis and/or hemorrhage are frequently found within pheochromocytomas, and acute hemorrhagic necrosis of the tumor may result in a massive catecholamine release $(2,10,19)$. The patient in the present case experienced abdominal pain on the night of the admission, and the urinary levels of adrenaline, noradrenaline, metanephrine, normetanephrine, and VMA peaked during the same time period. Moreover, the levels of adrenaline, noradrenaline and dopamine in the exudate of the necrotic lesion in the present case were evaluated. These results suggest that acute hemorrhagic tumor necrosis occurred on the night of admission and that the excess catecholamine release induced the patient's abdominal pain and reverse takotsubo cardiomyopathy on the day after admission.

The authors state that they have no Conflict of Interest (COI).

\section{References}

1. Lenders JW, Eisenhofer G, Mannelli M, Pacak K. Pheochromocytoma. Lancet 366: 665-675, 2005.

2. Braovo EL, Tarazi RC, Gifford RW, Stewart BH. Circulating and uarinary cathecholamines in pheochromocytoma. Diagnostic and pathophysiologic implications. N Eng J Med 301: 682-686, 1979.

3. Naderi N, Amin A, Setayesh A, Pouraliakbar H, Mozaffari K, Maleki M. Pheochromocytoma-induced reverse takotsubo with rapid recovery of left ventricular function. Cardiol J 19: 527-531, 2012.

4. Wu XM, Chen JJ, Wu CK, Lin LY, Tsung CD. Pheochromocytoma presenting as acute myocarditis with cardiogenic shock in two cases. Intern Med 47: 2151-2155, 2008.

5. Ueda T, Oka N, Matsumoto A, et al. Pheochromocytoma presenting as recurrent hypotension and syncope. Intern Med 44: 222227, 2005.

6. McEntee RK, Coyle D, Meyer M. Severe dilated cardiomyopathy treatment in an undiagnosed adrenal pheochromocytoma. Cric Heart Fail 4: e10-e12, 2011.
7. Baxter MA, Hunter P, Thompson GR, London DR. Pheochoromocytomas as a cause of hypotension. Clin Endocrinol 37: 304-306, 1992.

8. Iio K, Sakurai S, Kato T, et al. Endomyocardial biopsy in a patient with hemorrhage pheochromocytoma presenting as inverted takotsubo cardiomyopathy. Heart Vessels 28: 255-263, 2013.

9. Recalde AS, Costero O, Oliver JM, Iborra C, Ruiz E, Jose A. Phenochromocytoma-related cardiomyopathy: Inverted takotsubo contractile pattern. Circulation 113: e738-e739, 2006.

10. Kim S, Yu A, Filippone LA, Kolansky DM, Raina A. Invertedtakotubo pattern cardiomyopathy secondary to pheochromocytoma: A clinical case and literature review. Clin Cardiol 33: 200205, 2010.

11. Cho SK, Kim KH, Cho JY, et al. Pheochromocytoma as a rare hidden cause of inverted stress cardiomyopathy. J Cardoiovasc Ultrasoud 22: 80-83, 2014.

12. Kawai S, Suzuki H, Yamaguchi H, et al. Ampulla cardiomyopathy ('takotsubo' cardiomyopathy): Reversible left ventricular dysfunction with ST-segment elevation. Jpn Circ J 64: 156-159, 2000.

13. Bybee KA, Prasad A. Stress-related cardiomyopathy syndrome. Circulation 118: 397-409, 2008.

14. Song BG, Chun WJ, Park YH, et al. The clinical characteristics, laboratory parameters, electrocardiographic findings of reverse or inverted takotsubo cardiomyopathy: Comparison with mid or apical variant. Clin Cardiol 34: 693-699, 2011.

15. Kobayashi Y, Kobayashi Y, Hirohata A. Phenochromocytoma found in takotsubo cardiomyopathy patients. J Invasive Cardiol 26: E76-E77, 2014.

16. Ramaraj R, Movahed MR. Reverse or inverted takotsubo cardiomyopathy (reverse left ventricular apical ballooning syndrome) presents at a younger compared with the mid or apical variant and is always associated with triggering stress. Congest Heart Fail 16: 284-286, 2010.

17. Rossi AP, Bing-You RG, Thomas LR. Recurrent takotubo cardiomyopathy associated with pheochromocytoma. Endocr Pract 15: 560-562, 2009.

18. Chia P, Foo D. Takotsubo cardiomyopathy precipitated by pheochromocytoma crisis. Cardiol J 18: 564-567, 2011.

19. Mohamed HA, Aldakar MO, Habib N. Cardiogenic shock due to acute hemorrhagic necrosis of a pheochromocytoma: a case report and review of the literature. Can J Cardiol 19: 573-576, 2003.
(C) 2015 The Japanese Society of Internal Medicine http://www.naika.or.jp/imonline/index.html 